Sciendo

DOI: 10.2478/awutm-2018-0014
Analele Universităţii de Vest,

Timişoara

Seria Matematică - Informatică

LVI, 2, (2018), 28- 42

\title{
A stop over Jain operators and their generalizations
}

\author{
Octavian Agratini
}

Dedicated to Professor Mihail Megan on the occasion of his 70th birthday

\begin{abstract}
On the last five decades the interest of the study of positive approximation processes have emerged with growing evidence. A special place is occupied by the in-depth study of classical operators. The most eloquent example is Bernstein operator which represents a permanent challenge for the researches in the mentioned field.

However, in this synthesis we focused on presenting a class of operators introduced by G.C. Jain in the 1970s that have long been in a shadowy cone. In recent years many papers have appeared about their properties and many generalizations have been analyzed. In our approach, there is no question of an exhaustive treatment, but only of collecting some published results that prove the importance of this class through the generous possibilities offered by the approximation of signals from different function spaces.
\end{abstract}

AMS Subject Classification (2000). 41A36, 41A35, 41A25. Keywords. Linear positive operator, Poisson distribution, Korovkin theorem, modulus of smoothness, weighted space, statistical convergence, Voronovskaja theorem.

\section{Introduction}

Set $\mathbb{N}_{0}=\{0\} \cup \mathbb{N}$ and $e_{j}, j \in \mathbb{N}_{0}$, monomials of degree $j, e_{0}(x)=1, e_{j}(x)=x^{j}$, $j \in \mathbb{N}$. The starting point is represented by a Poisson-type distribution with 
two parameters given by

$$
w_{\beta}(k ; \alpha)=\frac{\alpha}{k !}(\alpha+k \beta)^{k-1} e^{-(\alpha+k \beta)}, k \in \mathbb{N}_{0},
$$

for $\alpha>0$ and $|\beta|<1$. By using Lagrange inversion, in [17, Lemma 1] was proved

$$
\sum_{k=0}^{\infty} w_{\beta}(k ; \alpha)=1
$$

In what follows, we consider $w_{\beta}(k ; 0)=\delta_{k, 0}$, here $\delta_{k, 0}$ meaning Kronecker's delta symbol. Jain [17] introduced and studied the following class of positive linear operators

$$
\left(P_{n}^{[\beta]} f\right)(x)=\sum_{k=0}^{\infty} w_{\beta}(k ; n x) f\left(\frac{k}{n}\right), x \geq 0,
$$

where $\beta \in[0,1)$ and $f \in C\left(\mathbb{R}_{+}\right)$whenever the above series is convergent. Due to relation (1.2), the operators have the property

$$
P_{n}^{[\beta]} e_{0}=e_{0}
$$

consequently, they reproduce the constants. Moreover, the following formulas occur [17, Eqs. (2.13)-(2.14)]

$$
P_{n}^{[\beta]} e_{1}=\frac{1}{1-\beta} e_{1}, P_{n}^{[\beta]} e_{2}=\frac{1}{(1-\beta)^{2}} e_{2}+\frac{1}{n(1-\beta)^{3}} e_{1} .
$$

Based on relations (1.4) and (1.5), the second central moment for $P_{n}^{[\beta]}$ operator is given by the relation

$$
\left(P_{n}^{[\beta]} \varphi_{x}^{2}\right)(x)=\left(\frac{\beta}{1-\beta}\right)^{2} x^{2}+\frac{x}{n(1-\beta)^{3}}, x \geq 0
$$

where $\varphi_{x}(t)=|t-x|,(t, x) \in \mathbb{R}_{+} \times \mathbb{R}_{+}$. Also, $P_{n}^{[\beta]}$ enjoys the interpolating property in $x=0$, i.e.,

$$
\left(P_{n}^{[\beta]} f\right)(x)=f(0) .
$$

In special case $\beta=0, P_{n}^{[0]}, n \in \mathbb{N}$, turn into well-known Szász-Mirakjan operators, see [26], [21],

$$
\left(P_{n}^{[0]} f\right)(x) \equiv\left(S_{n} f\right)(x)=e^{-n x} \sum_{k=0}^{\infty} \frac{(n x)^{k}}{k !} f\left(\frac{k}{n}\right), x \geq 0 .
$$


Studying relations (1.5) or the second central moment for Jain operators and appealing to Bohman-Korovkin theorem, it can be observed that the sequence $\left(P_{n}^{[\beta]}\right)_{n \geq 1}$ does not tend to the identity operator. To become an approximation process, we proceed as follows. For each $n \in \mathbb{N}$, the constant $\beta$ will be replaced by a number $\beta_{n} \in[0,1)$. If

$$
\lim _{n \rightarrow \infty} \beta_{n}=0 \text {, }
$$

then $\lim _{n \rightarrow \infty}\left(P_{n}^{\left[\beta_{n}\right]} e_{j}\right)(x)=e_{j}(x), j \in\{0,1,2\}$, uniformly on any compact interval $K \subset \mathbb{R}_{+}$. Consequently,

$$
\lim _{n \rightarrow \infty}\left(P_{n}^{\left[\beta_{n}\right]} f\right)(x)=f(x) \text {, uniformly in } x \in K .
$$

Due to the simple expression, over time, operators defined by (1.6) were the subject of countless papers. Instead, Jain operators have focused the researchers' attention especially in the last decade.

The purpose of this article is to give an overview of what has been recently investigated as regards Jain operators. The weak point of the work is the subjective selection of the obtained outcomes, with the author's assumed risk of not knowing all the notable published results related to this family of operators. However, we primarily focused on our own research on the subject.

In distinct sections we will present approximation properties related to discrete operators $P_{n}^{[\beta]}$ and $P_{n}^{\left[\beta_{n}\right]}$, respectively integral generalizations thereof. To achieve a fluent exposure, sometimes we have modified the notations found in various used papers. Another goal followed was that the presentation to be essentially self contained.

\section{Preliminaries}

We list the main notations and notions used throughout the paper.

$C_{B}\left(\mathbb{R}_{+}\right)$indicates the Banach space of all real-valued bounded and continuous functions defined on $\mathbb{R}_{+}$. The space is endowed with the sup-norm $\|\cdot\|$, where $\|f\|=\sup _{x \geq 0}|f(x)|, f \in C_{B}\left(\mathbb{R}_{+}\right)$. In connection with the estimation of the rate of convergence, the following moduli of smoothness play a basic role:

$$
\begin{aligned}
\omega_{1}(f ; \delta) & =\sup _{0 \leq h \leq \delta} \sup _{x \geq 0}|f(x+h)-f(x)| \\
& =\sup \left\{f(x)-f\left(x^{\prime}\right): x, x^{\prime} \in \mathbb{R}_{+},\left|x-x^{\prime}\right| \leq \delta\right\}, \\
\omega_{2}(f ; \delta) & =\sup _{0 \leq h \leq \delta} \sup _{x \geq 0}|f(x+2 h)-2 f(x+h)+f(x)|,
\end{aligned}
$$


where $f \in C_{B}\left(\mathbb{R}_{+}\right)$.

Another useful tool in estimating the rate of convergence of a sequence of operators to the function that is approximated is K-functional introduced by Peetre [23]. If $X_{0}, X_{1}$ are two Banach spaces with $X_{1}$ continuously embedded in $X_{0}\left(X_{1} \hookrightarrow X_{0}\right)$, the K-functional is defined for any $f \in X_{0}$ as follows

$$
K\left(f, \delta ; X_{0}, X_{1}\right) \equiv K(f ; \delta)=\inf _{g \in X_{1}}\left\{\|f-g\|_{X_{0}}+\delta\left\|g^{\prime \prime}\right\|_{X_{1}}\right\}, \delta \geq 0 .
$$

The space of all uniformly continuous real functions on $\mathbb{R}_{+}$is denoted by $U C\left(\mathbb{R}_{+}\right)$. Also, $C^{k}\left(\mathbb{R}_{+}\right), k \in \mathbb{N}$, denotes the space of all real-valued continuous functions on $\mathbb{R}_{+}$which are $k$ times continuously differentiable in $\mathbb{R}_{+}$. Because we are discussing about an approximation process associated with functions defined on unbounded intervals, it is natural to study it in weighted spaces, not by considering only bounded functions. In what follows we consider a one-to-one correspondence $\rho: \mathbb{R}_{+} \rightarrow \mathbb{R}_{+}$, continuously differentiable on the domain such that $\frac{d}{d x} \rho(x) \geq 1, x \in \mathbb{R}_{+}$. Consequently, $\rho(0)=0$ and $\lim _{x \rightarrow \infty} \rho(x)=\infty$ hold. Also, let the weight $\varphi$ be defined by $\varphi=e_{0}+\rho^{2}$. We consider the weighted space

$$
B_{\varphi}\left(\mathbb{R}_{+}\right)=\left\{f: \mathbb{R}_{+} \rightarrow \mathbb{R}:|f(x)| \leq M_{f} \varphi(x), x \in \mathbb{R}_{+}\right\},
$$

where $M_{f}$ is a positive constant depending only on $f$. This space can be endowed with the norm $\|\cdot\|_{\varphi}$, where

$$
\|f\|_{\varphi}=\sup _{x \geq 0} \frac{|f(x)|}{\varphi(x)} .
$$

Further, we denote the subspace of all continuous functions in $B_{\varphi}\left(\mathbb{R}_{+}\right)$by $C_{\varphi}\left(\mathbb{R}_{+}\right)$. Moreover, $C_{\varphi}^{*}\left(\mathbb{R}_{+}\right)$stands for the closed subspace of $C_{\varphi}\left(\mathbb{R}_{+}\right)$formed by the functions $f$ for which $\lim _{x \rightarrow \infty} f(x) / \varphi(x)$ exists and is finite.

It is worth mentioning that Bustamante and Morales de la Cruz [6] have shown that the theory of approximation by linear operators in the spaces $C_{\varphi}^{*}\left(\mathbb{R}_{+}\right)$can be reduced to the same theory for the space $C([0,1])$ of continuous functions on the interval $[0,1]$ endowed with the sup-norm.

In what follows we briefly recall the concept of statistical convergence. It, originally appeared in Steinhaus [25] and Fast [10] papers, is based on the notion of the density of subsets of $\mathbb{N}$ and it can be viewed as a regular method of summability of sequences. The density of a set $K \subset \mathbb{N}$ is defined by

$$
\delta(K)=\lim _{n \rightarrow \infty} \frac{1}{n} \sum_{k=1}^{n} \chi_{K}(k),
$$


provided the limit exists, where $\chi_{K}$ is the characteristic function of $K$. Actually, the sum of the right hand side represents the cardinality of the set $\{k \leq n: k \in K\}$. A sequence $x=\left(x_{k}\right)_{k \geq 1}$ is statistically convergent to a real number $L$, denoted $s t-\lim _{k \rightarrow \infty} x_{k}=L$, if, for every $\varepsilon>0$,

$$
\delta\left(\left\{k \in \mathbb{N}:\left|x_{k}-L\right| \geq \varepsilon\right\}\right)=0
$$

holds.

It is known that any convergent sequence is statistically convergent, but not conversely. To become reciprocal true, one can use the result set in 2000 by Fridy and Khan [11, Theorem 2.2]: if the sequence $x=\left(x_{n}\right)_{n \geq 1}$ satisfies

$$
s t-\lim _{n \rightarrow \infty} x_{n}=L \text { and } n \Delta x_{n+1} \geq-c,
$$

for some $c>0$ and for every $n \in \mathbb{N}$, then $\lim _{n \rightarrow \infty} x_{n}=L$, where $\Delta$ denotes the backward difference. We also mention, Connor [7] proved that statistical convergence is equivalent to the strong Cesàro summability in the space of all series with bounded elements. It can be concluded that this type of convergence can be viewed as a regular method of series summability.

The application of this notion to the study of positive linear operators was attempted only in 2002 by Gadjiev and Orhan [14] who obtained Korovkintype theorems via statistical convergence. Their main result says: if

$$
s t-\lim _{n \rightarrow \infty}\left\|L_{n} e_{j}-e_{j}\right\|_{[a, b]}=0 \text { for } j=0,1,2,
$$

then

$$
s t-\lim _{n \rightarrow \infty}\left\|L_{n} f-f\right\|_{[a, b]}=0,
$$

where $\left(L_{n}\right)_{n \geq 1}$ is a sequence of linear positive operators defined on $C(J)$ and the sup-norm $\|\cdot\|_{[a, b]}$ is taken on the compact $[a, b] \subset J$ (see $[14$, Theorem 1]).

Closely related to statistical convergence is A-statistical convergence, where $A=\left(a_{n, k}\right)$ is an infinite summability matrix. For a given sequence $x=$ $\left(x_{n}\right)_{n \geq 1}$, the A-transform of $x$ denoted by $A x=(A x)_{n \geq 1}$ is defined by

$$
(A x)_{n}=\sum_{k=1}^{\infty} a_{n, k} x_{k}, n \in \mathbb{N}
$$

provided the series converges for each $n$. Suppose that $A$ is a non-negative summability matrix, i.e., $a_{n, k} \geq 0$ and the matrix transformation of any 
convergent sequence preserves its limit. The sequence $x=\left(x_{n}\right)_{n \geq 1}$ is Astatistically convergent to the real number $L$ if, for every $\varepsilon>0$, one has

$$
\lim _{n \rightarrow \infty} \sum_{k \in I(\varepsilon)} a_{n, k}=0
$$

where $I(\varepsilon)=\left\{k \in \mathbb{N}:\left|x_{k}-L\right| \geq \varepsilon\right\}$. We write $s t_{A}-\lim _{n} x_{n}=L$, see, e.g., [12].

\section{Properties of the discrete operators}

We start the presentation by indicating a Voronovskaja type formula established in 2012 by Farcaş [9, Theorem 2.5]. The following identity

$$
\lim _{n \rightarrow \infty} n\left(\left(P_{n}^{\left[\beta_{n}\right]} f\right)(x)-f(x)\right)=\frac{x}{2} f^{\prime \prime}(x), x>0,
$$

takes place for $f \in C^{2}\left(\mathbb{R}_{+}\right)$, assuming both relation (1.7) and a more restrictive condition, namely $\lim _{n \rightarrow \infty} n \beta_{n}=0$.

The rate of convergence inferring the speed at which a convergent sequence approaches its limit, can be expressed using the first two moduli.

Theorem 3.1. ([2, Theorem 2]). Let $P_{n}^{[\beta]}, n \in \mathbb{N}$, be defined by (1.3). For every $f \in C_{B}\left(\mathbb{R}_{+}\right)$,

$$
\left|\left(P_{n}^{[\beta]} f\right)(x)-f(x)\right| \leq \omega_{1}\left(f ; \frac{\beta}{1-\beta} x\right)+c \omega_{2}\left(f ; \delta_{n} \sqrt{\max \left\{x, x^{2}\right\}}\right), x \geq 0,
$$

takes place, where $c$ is a certain positive constant and

$$
\delta_{n}=\frac{1}{2} \sqrt{\frac{\beta^{2}}{(1-\beta)^{2}}+\frac{1}{2 n(1-\beta)^{3}}} .
$$

Returning at (2.1), we consider

$$
X_{0}=C_{B}\left(\mathbb{R}_{+}\right) \text {and } X_{1}=C_{B}^{2}\left(\mathbb{R}_{+}\right)=\left\{g \in C_{B}\left(\mathbb{R}_{+}\right): g^{\prime}, g^{\prime \prime} \in C_{B}\left(\mathbb{R}_{+}\right)\right\},
$$

both spaces being endowed with the sup-norm. In comparison with the modulus of smoothness $\omega_{2}$, it takes place: the positive constants $c_{1}$ and $c_{2}$ exist such that

$$
c_{1} \omega_{2}(f ; \delta) \leq K\left(f ; \delta^{2}\right) \leq c_{2} \omega_{2}(f ; \delta), \delta \geq 0,
$$


see, for example, [18, Proposition 6.1].

Recalling that a function $f$ defined on $\mathbb{R}_{+}$is locally Lip $\alpha$ on $E(0<\alpha \leq 1$, $\left.E \subset \mathbb{R}_{+}\right)$if it satisfies the condition

$$
|f(x)-f(y)| \leq M_{f}|x-y|^{\alpha},(x, y) \in \mathbb{R}_{+} \times E,
$$

where $M_{f}$ is a constant depending only on $f$, we present a local approximation valid result for smooth functions.

Theorem 3.2. ([2, Theorem 1]). Let $P_{n}^{[\beta]}, n \in \mathbb{N}$, be defined by (1.3), $\alpha \in(0,1]$ and $E$ be a subset of $\mathbb{R}_{+}$. If $f$ is locally Lipa on $E$, then we get

$$
\left|\left(P_{n}^{[\beta]} f\right)(x)-f(x)\right| \leq M_{f}\left(c_{n}(\alpha, \beta) \max \left\{x^{\alpha / 2}, x^{\alpha}\right\}+2 d^{\alpha}(x, E)\right), x \geq 0,
$$

where

$$
c_{n}(\alpha, \beta)=\left(\frac{\beta}{1-\beta}\right)^{\alpha}+\left(\frac{1}{n(1-\beta)^{3}}\right)^{\alpha / 2}
$$

and $d(x, E)$ is the distance between $x$ and $E$ defined as

$$
d(x, E)=\inf \{|x-y|: y \in E\} .
$$

Also, the statistical convergence of Jain operators was studied.

Theorem 3.3. ([2, Theorem 3]). Let $\beta_{n} \in[0,1)$ and $P_{n}^{\left[\beta_{n}\right]}, n \in \mathbb{N}$, be given as in (1.3). If $[a, b] \subset \mathbb{R}_{+}$and

$$
s t-\lim _{n \rightarrow \infty} \beta_{n}=0,
$$

then, for any $f \in C\left(\mathbb{R}_{+}\right)$, we have

$$
s t-\lim _{n}\left\|P_{n}^{\left[\beta_{n}\right]} f-f\right\|_{[a, b]}=0 .
$$

Using function $\rho$ presented by us in the previous section that enjoys the properties mentioned there, Olgun, Taşdelen and Erençin [22] defined and studied the following generalization of Jain operators

$$
\left(P_{n}^{[\beta, \rho]} f\right)(x)=\sum_{k=0}^{\infty} f\left(\rho^{-1}\left(\frac{k}{n}\right)\right) w_{\beta}(k ; n \rho(x)), x \geq 0 .
$$

This class is useful in approximating the functions of weighted spaces, more precisely takes place 
Theorem 3.4. ([22, Theorem 2.3]). Let $\beta_{n} \in[0,1)$ such that (1.7) holds, and $\left(P_{n}^{\left[\beta_{n}, \rho\right]}\right)_{n \geq 1}$ be given as in (3.2). Then, for any $f \in C_{\varphi}^{*}\left(\mathbb{R}_{+}\right)$, we have

$$
\lim _{n \rightarrow \infty}\left\|P_{n}^{\left[\beta_{n}, \rho\right]} f-f\right\|_{\varphi}=0
$$

Also, a Voronovskaja type theorem was established.

Theorem 3.5. ([22, Theorem 3.1]). Let $\beta_{n} \in[0,1)$ such that $\lim _{n \rightarrow \infty} \beta_{n}=0$ and $\lim _{n \rightarrow \infty} n \beta_{n}=0$. Let $f \in C\left(\mathbb{R}_{+}\right), x \in \mathbb{R}_{+}$, and suppose that the first and second derivatives of $f \circ \rho^{-1}$ exist at $\rho(x)$. If $f \circ \rho^{-1} \in B\left(\mathbb{R}_{+}\right)$, then we have

$$
\lim _{n \rightarrow \infty} n\left(\left(P_{n}^{\left[\beta_{n}, \rho\right]} f\right)(x)-f(x)\right)=\frac{\rho(x)}{2}\left(f \circ \rho^{-1}\right)^{\prime \prime}(\rho(x)) .
$$

By choosing $\rho$ as the identity map on $\mathbb{R}_{+}$, the conditions required for it in Preliminaries are met and identity (3.3) turns into (3.1).

In the paper [1], the authors have been able to achieve a complete asymptotic expansion for the sequence $\left(P_{n}^{\left[\beta_{n}\right]}\right)_{n \geq 1}$. In order to present in detail the established formula, at first step we will inventory the used coefficients.

We recall $\sigma(n, k)$, the Stirling numbers of the second kind given by explicit formula

$$
\sigma(n, k)=\frac{1}{k !} \sum_{i=0}^{k}(-1)^{i}\left(\begin{array}{l}
k \\
i
\end{array}\right)(k-i)^{n}
$$

these numbers counting the number of ways to partition a set of $n$ labeled objects into $k$ non-empty unlabeled subsets. On the other hand, we consider the function $g$ defined on $\mathbb{C}$ by analytic continuation, where, for $z \neq 0$, this function is given as follows

$$
g(z)=\frac{1+z-e^{\beta z}}{z e^{\beta z}}
$$

Hence, it has the power-series expansion

$$
g(z)=\sum_{\nu=0}^{\infty}\left(1-\frac{\beta}{\nu+1}\right) \frac{(-\beta z)^{\nu}}{\nu !}, z \in \mathbb{C} .
$$

Set

$$
c_{l, j}(\beta)=\left.\left(\frac{\partial}{\partial z}\right)^{j} g^{-l}(z)\right|_{z=0} .
$$

With the help of (3.4) and (3.5), we define the coefficients

$$
b_{k, s}(\beta)=\sum_{l=0}^{k} \sum_{r=k+1}^{s}(-1)^{s-r}\left(\begin{array}{l}
s \\
r
\end{array}\right) \sigma(r, r-l)\left(\begin{array}{c}
r-l-1 \\
k-l
\end{array}\right) c_{r-l, k-l}(\beta),
$$


where $s \in \mathbb{N}$. All sums are to be read as null if the lower index exceeds the upper one.

For $r \in \mathbb{N}, \alpha>0$ and $x \in(0, \infty)$. let $W^{\alpha}[r ; x]$ be the class of functions on $(0, \infty)$ satisfying the growth condition $f(t)=\mathcal{O}\left(t^{\alpha}\right)$ as $t \rightarrow \infty$, which admit a derivative of order $r$ at the point $x$.

Theorem 3.6. ([1, Theorem 1]). Let $q \in \mathbb{N}$ and $x>0$. Furthermore, let $\left(\beta_{n}\right)$ be a sequence of real numbers with $0 \leq \beta_{n}<1$ and $\beta_{n}=\mathcal{O}\left(n^{-1 / 2}\right)$ as $n \rightarrow \infty$. Then, for each function $f \in W^{2 q}[r ; x]$, the Jain operators possess the asymptotic expansion

$$
\left(P_{n}^{\left[\beta_{n}\right]} f\right)(x)=f(x)+\sum_{s=1}^{2 q} \frac{f^{(s)}(x)}{s !}\left(\frac{\beta_{n} x}{1-\beta_{n}}\right)^{s}+\sum_{k=1}^{q} a_{k}^{\left[\beta_{n}\right]}(f, x) n^{-k}+o\left(n^{-q}\right)
$$

as $n \rightarrow \infty$, where the coefficients $a_{k}^{\left[\beta_{n}\right]}(f, x)$ are given by

$$
a_{k}^{\left[\beta_{n}\right]}(f, x)=\sum_{s=0}^{2 q} \frac{f^{(s)}(x)}{s !} x^{s-k} b_{k, s}\left(\beta_{n}\right)
$$

and $b_{k, s}\left(\beta_{n}\right)$ is as defined in (3.6).

In the same paper cited, a new Voronovskaja-type result was obtained. Assuming that the limit

$$
\lim _{n \rightarrow \infty} \sqrt{n} \beta_{n}=\gamma
$$

exists with a finite value, under the conditions of previous theorem, for each function $f \in W^{2}[r ; x]$ one has

$$
\lim _{n \rightarrow \infty} \sqrt{n}\left(\left(P_{n}^{\left[\beta_{n}\right]} f\right)(x)-f(x)\right)=\gamma x f^{\prime}(x), x>0 .
$$

Moreover, if the limit

$$
\lim _{n \rightarrow \infty} n \beta_{n}=\gamma
$$

exists with a finite value, we have

$$
\lim _{n \rightarrow \infty} n\left(\left(P_{n}^{\left[\beta_{n}\right]} f\right)(x)-f(x)\right)=\gamma x f^{\prime}(x)+\frac{1}{2} x f^{\prime \prime}(x), x>0 .
$$

Again, for $\gamma=0$ we reobtain (3.1).

Finally we mention a very recent paper [5] in which the authors generalize the classical Lupaş operators [19] using Jain's base defined in (1.1). Among the established results, they proved [5, Theorem 4.1] that the new construction has the monotony property namely: if $f$ is a convex function on $\mathbb{R}_{+}$, then the sequence of operators which approximates $f$ is non-decreasing. 


\section{Integral modifications of Jain operators}

Various generalizations of Jain discrete operators are known. Best of our knowledge, the first approach was achieved by Uman and Razi in 1985 who defined a Kantorovich-type integral modification expressed as follows [28, Eq. (2.12)]

$$
\left(K_{n}^{[\beta]} f\right)(x)=n \sum_{k=0}^{\infty} w_{\beta}(k ; n x) \int_{\frac{k}{n}}^{\frac{k+1}{n}} f(t) d t,
$$

where $f$ is locally integrable function and the right hand side of relation (4.1) is finite.

Another integral variant was introduced and studied by Tarabie [27]. The operators have been called Jain-Beta and defined by the following relation

$$
\left(J_{n}^{[\beta]} f\right)(x)=\sum_{k=1}^{\infty} \frac{w_{\beta}(k ; n x)}{B(n+1, k)} \int_{0}^{\infty} f(t) \frac{t^{k-1}}{(1+t)^{n+k+1}} d t+e^{-n x} f(0),
$$

$x \geq 0$, where $n \geq 2$ and $f \in C_{\varphi}\left(\mathbb{R}_{+}\right)$with $\varphi=e_{0}+e_{1}^{2}$. These operators have Jain and Beta basis functions in summation and integration, respectively. For $\beta=0$ the operators $J_{n}^{[0]}$ reduce to the mixed Szász-Beta operators investigated earlier by Gupta and Noor [16].

Among the approximation properties highlighted on $J_{n}^{\left[\beta_{n}\right]}(n \geq 2)$ operators, we enunciate

Theorem 4.1. ([27, Theorem 4]). Let $A=\left(a_{n, k}\right)$ be a non-negative regular summability matrix and $\lambda>0$ be fixed. Let $J_{n}^{\left[\beta_{n}\right]}, n \geq 2$, be defined as in (4.2), where $\left(\beta_{n}\right)_{n \geq 2}, 0 \leq \beta_{n}<1$, satisfies

$$
s t_{A}-\lim _{n \rightarrow \infty} \beta_{n}=0 .
$$

We get

$$
s t_{A}-\lim _{n \rightarrow \infty}\left\|J_{n}^{\left[\beta_{n}\right]} f-f\right\|_{\varphi_{\lambda}}=0, f \in C_{\varphi_{0}}\left(\mathbb{R}_{+}\right),
$$

where $\varphi_{\lambda}=e_{0}+e_{1}^{2+\lambda}, \varphi_{0}=e_{0}+e_{1}^{2}$.

Further, we present an extension in Durrmeyer sense of $P_{n}^{[\beta]}$ operators which has appeared in the paper [3]. These operators are designed as follows

$$
\begin{gathered}
\left(\Lambda_{n}^{[\beta]} f\right)(x)=e^{-n x} f(0) \\
+\frac{n}{1-\beta} \sum_{k=1}^{\infty} \frac{w_{\beta}(k ; n x)}{\Gamma(k)} \int_{0}^{\infty} e^{-n t /(1-\beta)}\left(\frac{n t}{1-\beta}\right)^{k-1} f(t) d t
\end{gathered}
$$


where $f \in \mathcal{F}\left(\mathbb{R}_{+}\right)$, the space of all functions $f: \mathbb{R}_{+} \rightarrow \mathbb{R}$ which are integrable on each compact interval on $\mathbb{R}_{+}$and for which the relation $\Lambda_{n}^{[\beta]}|f|<\infty$ occurs.

For the special case $\beta=0$, from (4.3) Phillips operators [24] are recovered. By $\Lambda_{n}^{[\beta]}, n \geq 1$, the affine functions are fixed points of these operators, property that we do not meet in constructions presented at (4.1) and (4.2). Moreover, for any $b>0$ fixed we have

$$
\lim _{n \rightarrow \infty} \Lambda_{n}^{[\beta]} f=f \text { uniformly on }[0, b],
$$

provided $f \in C\left(\mathbb{R}_{+}\right) \cap \mathcal{F}\left(\mathbb{R}_{+}\right)$. It is interesting to note that for a fixed $0<\beta<1$, the genuine Jain operators do not form an approximation process, instead our integral generalization has this feature.

It is known that several classic positive approximation processes can be reintroduced by using concepts of probability theory. Following this path, the operators defined by (4.3) can be redesigned. We briefly present this approach.

Let $X=\left\{X_{t}: t \geq 0\right\}$ be a stochastic process starting at zero with independent stationary increments and, for each $t>0, X_{t}$ having a Gamma-type distribution with the density

$$
\rho_{t}(\beta ; u)=\frac{1}{(1-\beta) \Gamma(t)}\left(\frac{u}{1-\beta}\right)^{t-1} \exp \left(-\frac{u}{1-\beta}\right) \mathbf{1}_{\mathbb{R}_{+}^{*}}(u),
$$

where $\mathbf{1}_{\mathbb{R}_{+}^{*}}$ stands for the characteristic function of the set $\mathbb{R}_{+}^{*}$.

Let $Y=\{Y(u): u \geq 0\}$ be a Poisson-type process independent of $X$ and defined on the same probability space. For each $u \geq 0, Y(u)$ has the distribution

$$
P(Y(u)=k)=w_{\beta}(k ; u), k \in \mathbb{N}_{0},
$$

where $w_{\beta}(k ; u)$ is the same as in (1.1). With these data, replacing $n \in \mathbb{N}$ by $t>0$, the operators $\Lambda_{t}^{[\beta]}$ can be represented by the relation

$$
\left(\Lambda_{t}^{[\beta]} f\right)(x)=E\left[f\left(t^{-1} X_{Y(t x)}\right)\right],
$$

where $E$ denotes mathematical expectation.

Forwards we are studying another property of $\Lambda_{n}^{[\beta]}$ operators. From the point of view of approximation theory, the sequence of operators associated to a function $f$ must converge to approximated element. Usually, for continuous functions defined on a compact, uniform convergence takes place but for continuous functions defined on an unbounded interval only pointwise convergence occurs. We focus our attention on identifying functions for which these operators provide uniform convergence on $\mathbb{R}_{+}$. A result in this direction can be read further. 
Theorem 4.2. ([3, Theorem 3]). Let $\Lambda_{n}^{[\beta]}, n \in \mathbb{N}$, be defined by (4.3). Let $f \in C_{B}\left(\mathbb{R}_{+}\right)$and $f^{*}=f \circ e_{2}$. If $f^{*} \in U C\left(\mathbb{R}_{+}\right)$, then $\left(\Lambda_{n}^{[\beta]}\right)_{n \geq 1}$ converges uniformly to $f$ on $\mathbb{R}_{+}$as $n$ tends to infinity.

It worth mentioning a previous concern in this direction. For discrete SzászMirakjan operators defined at (1.6), a similar result was first established in [20, Theorem 1]. Also, for integral form with $\beta=0$, i.e. Phillips-MazharTotik operators, the study of the uniform convergence for $\left(\Lambda_{t}^{[0]} f\right)_{t>0}$ to $f$ on $\mathbb{R}_{+}$was achieved in the same paper [20, Theorem 3].

We recall that for a general class of linear positive approximation process designed using series, in [4] have been identified functions for which these operators provide uniform approximation over unbounded intervals. Among the special cases are included genuine Jain operators.

The behavior of $\Lambda_{n}^{[\beta]}$ operators on weighted space $C_{\varphi}^{*}\left(\mathbb{R}_{+}\right)$specified in the Preliminaries is described in the following

Theorem 4.3. ([3, Theorem 4]). Let $\Lambda_{n}^{[\beta]}, n \in \mathbb{N}$, be defined by (4.3). The following identity

$$
\lim _{n \rightarrow \infty}\left\|\Lambda_{n}^{[\beta]} f-f\right\|_{\varphi}=0, f \in C_{\varphi}^{*}\left(\mathbb{R}_{+}\right),
$$

holds, where $\varphi=e_{0}+e_{1}^{2}$.

In fact, relation (4.4) takes place for a general polynomial weight of the form $\varphi=e_{0}+e_{m}^{2}, m \in \mathbb{N}$ fixed. The assertion is based on the one hand on a result due to Gadzhiev [13, Theorem 2] and on the other hand by the expression of the operator's moments [3, Lemma 1] given by

$$
\left(\Lambda_{n}^{[\beta]} e_{j}\right)(x)=(1-\beta)^{j} \sum_{k=1}^{\infty} w_{\beta}(k ; n x) q_{j}\left(\frac{k}{n}\right), j \in \mathbb{N},
$$

where

$$
q_{j}(x)=\prod_{s=0}^{j-1}\left(x+\frac{s}{n}\right), x \geq 0 .
$$

Keeping the same weighted space, investigating deeper Jain-Kantorovich operators, Deniz recently established a Voronovskaja theorem in quantitative form [8, Corollary 1] which we present in the following

Theorem 4.4. Let $K_{n}^{\left[\beta_{n}\right]}, n \in \mathbb{N}$, be defined by (4.1) and $\beta_{n} \in[0,1)$ such that $\beta_{n}=o\left(n^{-1}\right)$ as $n$ tends to infinity.

For any function $f \in C_{\varphi}^{*}\left(\mathbb{R}_{+}\right), \varphi=e_{0}+e_{1}^{2}$, which admits a derivative of second order at the point $x>0$, we have

$$
\lim _{n \rightarrow \infty} n\left(\left(K_{n}^{\left[\beta_{n}\right]} f\right)(x)-f(x)\right)=\frac{1}{2}\left(f^{\prime}(x)+x f^{\prime \prime}(x)\right) .
$$


It is noteworthy that, using the functions $\omega_{\beta}(k ; \cdot)$ defined by $(1.1)$, in the monograph of Gupta and Agarwal [15, pages 60-63] we find the construction of a general integral class of linear positive operators in Durrmeyer's sense. This family contains as special cases Phillips operators and Szász-Beta type operators, see [16].

\section{References}

[1] U. Abel, O. Agratini, Asymptotic behaviour of Jain operators, $\mathrm{Nu}$ mer. Algor. 71 (2016), 553-565.

[2] O. Agratini, Approximation properties of a class of linear operators, Math. Meth. Appl. Sci. 36 (2013), 2353-2358.

[3] O. Agratini, On an approximation process of integral type, Appl. Math. Comp. 236 (2014), 195-201.

[4] O. Agratini, Uniform approximation of some classes of linear positive operators expressed by series, Applicable Analysis 94 (2015), No. 8, 1662-1669.

[5] G. Başcanbaz-Tunca, M. Bodur, D. Söylemez, On Lupaş-Jain operators, Stud. Univ. Babeş-Bolyai Math. 63 (2018), No. 4, 525-537.

[6] J. Bustamante, L. Morales de la Cruz, Korovkin type theorems for weighted approximation, Int. Journal of Math. Analysis 1 (2007), No. $26,1273-1283$.

[7] J.S. Connor, The statistical and strong p-Cesàro convergence of sequences, Analysis 8 (1988), 47-63.

[8] E. Deniz, Quantitative estimates for Jain-Kantorovich operators, Commun. Fac. Sci. Univ. Ank. Sér. A1 Math. Stat. 65 (2016), No. 2, 121-132.

[9] A. Farcaş, An aymptotic formula for Jain's operators, Stud. Univ. Babeş-Bolyai Math. 57 (2012), No. 4, 511-517.

[10] H. Fast, Sur la convergence statistique, Colloq. Math. 2 (1951), 241-244.

[11] J.A. Fridy, M.K. Khan, Statistical extensions of some classical Tauberian theorems, Proceedings of American Math. Soc. 128 (2000), No. 8, 2347-2355. 
[12] J.A. Fridy, H.I. Miller, A matrix characterization of statistical convergence, Analysis 11 (1991), 59-66.

[13] A.D. Gadzhiev, Theorems of Korovkin type, Math. Notes 20 (1976), No. 5, 995-998.

[14] A.D. Gadjiev, C. Orhan, Some approximation theorems via statistical convergence, Rocky Mountain J. Math. 32 (2002), No. 1, 129-138.

[15] V. Gupta, R.P. Agarwal, Convergence Estimates in Approximation Theory, Springer, 2014.

[16] V. Gupta, M.A. Noor, Convergence of derivatives for certain mixed Szász-Beta operators, J. Math. Anal. Appl. 321 (2006), 1-9.

[17] G.C. Jain, Approximation of functions by a new class of linear operators, J. Australian Math. Soc. 13 (1972), No. 3, 271-276.

[18] H. Johnen, Inequalities connected with moduli of smoothness, Matematicki Vesnik 9 (24) (1972), 289-303.

[19] A. Lupas, The approximation by some positive linear operators, In: Proceedings of the International Dortmund Meeting on Approximation Theory, (M.W. Müller et al., eds.), Mathematical Research, Akademie Verlag, Berlin, 86 (1995), 201-229.

[20] S.M. Mazhar, V. Totik, Approximation by modified Szász operators, Acta Sci. Math. 49 (1985), 257-269.

[21] G.M. Mirakjan, Approximation of functions with the aid of polynomials, Dokl. Akad. Nauk SSSR 31 (1941), 201-205 (in Russian).

[22] A. Olgun, F. Taşdelen, A. Erençin, A generalization of Jain's operators, Appl. Math. Comp. 266 (2015), 6-11.

[23] J. Peetre, A Theory of Interpolation of Normed Spaces, Notas de Matematica Instituto de Matemática Pura e Aplicada, Rio de Janeiro, 39 (1968), 1-86.

[24] R.S. Phillips, An inversion formula for Laplace transforms and semigroups of linear operators, Ann. Math. Second Ser. 59 (1954), 325-356.

[25] H. Steinhaus, Sur la convergence ordinaire et la convergence asymptotique, Colloq. Math. 2 (1951), 73-74. 
[26] O. Szász, Generalization of S. Bernstein's polynomials to the infinite interval, J. Res. Nat. Bur. Standards 45 (1950), 239-245.

[27] S. Tarabie, On Jain-Beta linear operators, Appl. Math. Inf. Sci. 6 (2012), No. 2, 213-216.

[28] S. Umar, Q. Razi, Approximation of function by generalized Szász operators, Commun. Fac. Sci. de l'Université d'Ankara, Serie $A_{1}$ : Mathématique 34 (1985), 45-52.

Octavian Agratini

Babeş-Bolyai University

Faculty of Mathematics and Computer Science

Str. Kogălniceanu, 1, 400084 Cluj-Napoca, Romania

Tiberiu Popoviciu Institute of Numerical Analysis

Romanian Academy

Str. Fântânele, 57, 400320 Cluj-Napoca, Romania

E-mail: agratini@math.ubbcluj.ro 